\title{
Algunas reflexiones sobre la "falacia naturalista". (¿Pueden tener contenidos normativos implícitos cierto tipo de juicios empíricos?)
}

\author{
ENRIQUE DUSSEL \\ Departamento de Filosofía \\ Universidad Autónoma Metropolitana-Iztapalapa
}

dussamb@servidor.unam.mx

\begin{abstract}
Resumen: Desde una reinterpretación de la posición de Hume, pasando por Moore y la lógica analítica, se sitúa el lugar teórico de la validez de la crítica a la "falacia naturalista" al mostrar que no se cae en ella cuando la premisa mayor de un argumento contiene enunciados descriptivos sobre la vida humana en cuanto humana; en este caso son "implícitamente" normativos. Explicitar la normatividad implícita contenida en el enunciado descriptivo es un modo de fundamentar (o mostrar en su fundamento) la normatividad del enunciado de actos humanos, sobre los que se tiene obligación ética en cuanto fundados en la responsabilidad originaria con respecto a la vida misma del sujeto ético.
\end{abstract}

PALABRAS ClaVE: falacia, ética, naturalista, principios.

La cuestión de la "falacia naturalista" recubre al menos cuatro dimensiones frecuentemente confundidas: I.) el complejo texto de Hume, en el que se sugiere la diferencia entre el "es" y el "deber-ser" dentro de la problemática propia de su época histórica; II.) la errada identificación de las propiedades "naturales" (lo deseable, útil o que produce felicidad) con las propiamente "éticas" (la bondad o el bien) analizada por George Moore, y que podría ser llamada la falacia de reducción; III.) la cuestión metaética desde el horizonte lógico-formal (desde Frege, el primer Wittgenstein, Ayer, etc.) que muestra la imposibilidad de la deducción analítica del "deber-ser" (juicio normativo) del "ser" (juicio empírico) o la "falacia naturalista" propiamente dicha; y IV.) la cuestión ética práctico-material que problematiza los diversos modos en que pueda efectuarse la descripción del "pasar", por "explicitación", desde alguna dimensión del "ser humano" (enunciado descriptivo) al "deber-ser" ético (enunciado normativo). Veamos resumidamente estos cuatro aspectos. 


\section{El COMPLEJO TEXTO DE DAVID HumE ${ }^{1}$}

La interpretación estándar, en la primera mitad del siglo xx desde el positivismo lógico hegemónico en filosofía, consistió en aceptar que Hume se refería explícitamente a la "falacia naturalista" tal como la definía la lógica formal (sección III. de las presentes reflexiones). Por el contrario, el historiador de la ética, A. MacIntyre, con el que discutí el punto cuando yo enseñaba como visiting professor en Duke University, sitúa la cuestión en su tiempo y contexto, y pone en duda dicha interpretación estándar. Las razones son varias. Recordemos algunas. Se indica que Hume no pudo referirse a la "falacia naturalista" en sentido estricto, porque los descubrimientos de la lógica formal sólo se realizarían a finales del siglo xIx y comienzos del xx. Además, en todo el tratamiento de las cuestiones éticas Hume parte del "ser" del ser humano (to be) en el nivel de los sentimientos, pasiones, placer o felicidad para, con referencia a ellos, situar los problemas éticos del "deber-ser" (ought to be). Por encontrarse en un momento prekantiano, no presupone todavía el dualismo del enfrentamiento del nivel material de la ética (en el nivel de los sentimientos) con la formalidad de su validez. ${ }^{2}$ Hume se habría contradicho si hubiera afirmado, en el complejo texto que citamos a continuación, la imposibilidad de pasar del "ser" al "deber-ser". Esta contradicción es evidente ya que Hume recurre continuamente a dicho pasaje (de los sentimientos a las exigencias éticas). La interpretación estándar presupone esta contradicción sólo en este texto, lo cual, a priori, parece poco plausible en un filósofo tan coherente como Hume. El texto es difícil, y aun confuso lingüísticamente, y por ello toda interpretación tendrá sus dificultades. Citémoslo:

Me sorprende que, en lugar de las cópulas o proposiciones es (is) y no es, no encuentro ninguna proposición que no esté conectada con un debería (ought) o no debería. Este cambio es imperceptible, pero es, sin embargo, la última consecuencia. Ya que este debería o no debería expresar una relación o afirmación nueva, y esto debería ser observado o explicado (explained), y debería al mismo tiempo darse alguna razón por lo que parece inconcebible (inconceivable) del

1 Véanse sobre Hume los trabajos de Atkinson 1964, MacIntyre 1964, Hudson 1964 y Beck 1974. Sobre la "falacia naturalista" en general Hunter 1962, Dubais 1967, Rabossi 1979, pp. 83 ss., Camps 1976, pp. 156-157, Sádaba 1989, pp. 212-216. Véase además desde la ética discursiva a Apel 1973, t. 2, pp. 362 ss., Habermas 1983, pp. 60 ss. Desde una interpretación histórica: MacIntyre 1981, pp. 12 ss. Considérese en mi Ética de la liberación (1998) en el "Índice alfabético de temas" los términos "falacia naturalista".

2 Véase este aspecto en el [117-121] de mi Ética de la liberación, 1998, pp. 169 ss., ya nombrada. 
todo, cómo esta nueva relación puede ser una deducción (deduction) de otras, que es completamente diferente de ella. ${ }^{3}$

Existen dos cuestiones centrales que hay que explicar. En primer lugar, que lo que no puede concebirse ("inconcebible [inconceivable]") no es que no pueda ser válido ese "pasaje" de un nivel a otro, sino que no se haya "de hecho e históricamente" tomado conciencia explícita del problema (que no se lo haya pensado hasta ese momento), porque se atribuía con anterioridad a Dios tanto el "ser" (por ser el creador de la naturaleza) como el "deberser" (por promulgar los mandatos). Que no se haya tomado conciencia sería explicable, porque "este cambio es imperceptible". Esto significa, al menos, que Hume sí está tomando conciencia de que se trata de una "nueva relación" (la "validez" normativa no se confunde ya con la mera "existencia" de las pasiones, emociones, etc.). Pero, y en segundo lugar, en el contexto histórico y de la obra misma de Hume, "deducción (deduction)" no puede tener el sentido analítico de "deducción (entailment)", ${ }^{4}$ sino de "inferencia" -en un sentido muy amplio como "pasaje" de un término a otro. MacIntyre concluye que "la reintepretación de este pasaje de Hume nos permite captar toda la cuestión de la razón práctica de una manera más creativa que lo que la tradición formalista permite". ${ }^{5}$ Si ésta muy plausible interpretación histórica fuera adecuada, no sólo Hume no hablaría de la "falacia naturalista" (en sentido estricto, como en la sección III, más adelante), por el contrario, nos está diciendo que el "deber-ser (ought)" ético se infiere o fundamenta en el "es (is)", entendido éste en el nivel de los sentimientos, el placer, la felicidad, las pasiones; pero, por otra parte, negando explícitamente una fundamentación racional ${ }^{6}$ o religiosa (como en el caso de la obediencia a

3 Hume 1958, libro III, parte I, sec. 1, p. 469: "I am surpriz'd to find, that instead of the usual copulations of propositions, is and is not, I meet with no proposition that is not connected with an ought, or an ought not. This change is imperceptible; but is, however, of the last consequence. For as this ought or ought not, expresses some new relation or affirmation, 'tis necessary that is should be observ'd and explain'd; and at the same time that a reason should be given, for what seems altogether inconceivable, how this new relation can be a deduction from others, which are entirely different from it."

4 Véase MacIntyre, 1964, p. 43: "Deduction [en Hume] therefor must mean inference and cannot mean entailment [...] What he [Hume] does is to ask how and if moral rules may be inferred from factual statements, and in the rest of book III of the Treatise he provides an answer to his own question" (p. 44), con un rotundo "si'" se infiere.

${ }^{5}$ Ibid., p. 45 ("the reinterpretation of this passage of Hume allows us to take up the whole question of practical reasoning in more fruitful way than the formalist tradition in ethics allow").

${ }^{6}$ Escribe Hume: "Morals excite passions, and produce or prevent actions. Reason of itself is utterly impotent in this particular. The rule of morality therefore, are not conclusions of our reason" (Ibid., p. 457). Nótese que la razón no puede pasar del "ser" al "deber-ser" porque 
los mandatos divinos). ${ }^{7}$ Era una muy crítica fundamentación secularizante (desde los sentimientos, material entonces), pero no formalista. Esto nos sirve de introducción a las siguientes reflexiones.

\section{LA DIFERENCIACIÓN DE PROPIEDADES EN GEORGE MOORE ${ }^{8}$}

Moore, en cambio, se encuentra ya inmerso en una reflexión ética que desde hacía más de un siglo se había habituado a diferenciar (y oponer) el nivel formal (sea de la validez o de la filosofía del lenguaje naciente) del material (reductivamente concebido como "naturalismo", en el caso de Hume en un nivel de los sentimientos). Sin embargo, cuando Moore se refiere a la "falacia naturalista" tampoco tendrá el significado posterior de la metaética analítica del lenguaje (ya que de ninguna manera se referirá al problema de la imposibilidad de la "deducción" del orden del "deber-ser" del orden del "ser"). Su investigación se centra en otra cuestión, por lo que, aunque use el término "falacia naturalista", no tendrá el mismo significado que en la lógica formal o la metaética del lenguaje posteriores.

Para Moore la "ética naturalista" (Naturalistic Ethics) ${ }^{10}$ no distingue entre propiedades naturales (natural properties) de objetos naturales (como el placer o lo que produce placer, el deseo o lo que es deseable, etc.) y las propiamente éticas (como la bondad o el bien). Si se confunden propiedades naturales con otras que son estrictamente éticas se puede caer en una "falacia naturalista". ${ }^{11}$ Herbert Spencer, con su vitalismo evolucionista, comete para Moore la falacia naturalista, en especial cuando piensa que lo que es una mediación (que se siente como placer) para producir una "vida des-

simplemente en este nivel no tiene nada que aportar. Son las pasiones, los sentimientos (el "ser" afectivo) los que permiten pasar del "ser" al "deber-ser", y esto es necesario y coherente en el pensamiento de Hume.

7 Es decir, Hume no puede oponerse a una ética material sustancial de la afectividad (en la cual se encuentra completamente inscrito), sino a una ética religiosa fundada en los designios divinos: "Hume is in fact repudiating a religious foundation for morality and putting in this place a foundation in human needs, interests, desires, and happiness" (MacIntyre, op. cit., p. 46). La interpretación estándar posterior parece que proyectó al pasado una posición histórica imposible para la época de Hume (error frecuente en el formalismo sin sentido histórico, y que cae inadvertidamente en otra falacia, la del "reduccionismo formalista").

8 Véase Schlipp 1942, Stevenson 1963, pp. 117-132; Blegvad 1963 y Lewy 1964.

9 Moore en Principia ethica (1903) se refiere a las cuestiones de la "falacia naturalista" al menos en su Prefacio (indirectamente), como introducción al tema (§§10-14); ante las éticas naturalistas ( $\S 25-34)$ y ante el hedonismo ( $\S 36-64)$. Véanse en el Index los lugares en que aparece puntualmente el tema "naturalistic fallacy" (p. 230).

10 Moore 1903, n. 11, p. 12 y todo el cap. 2 (nn. 24 ss.; pp. 37 ss.).

11 Ibid., n. 12, p. 13. 
arrollada" (increased life) ${ }^{12}$ debe considerarse "bien". De la misma manera, el hedonismo cae en la falacia al confundir el mero "placer" con "bien". ${ }^{13}$ Por nuestra parte podemos indicar que, en efecto, no deben confundirse las propiedades naturales con las éticas, pero que los presupuestos de Moore - su ingenua axiología metafísica y la ambigüedad de exponer que el "bien" es indefinible (lo que prueba la falta de un marco teórico suficientemente complejo) - lo hacen cometer una "falacia reduccionista", al no distinguir entre la referencia de los enunciados descriptivos (dirigida teóricamente a objetos del mundo) de los normativos (dirigida prácticamente a exigencias del sujeto humano situado en una estructura intersubjetiva, cultural, histórica). Por ello, Moore no puede analizar tampoco la diferencia entre diversos tipos de pretensiones, ya que la "pretensión de verdad teórica"14 de los primeros no se identifica con la "pretensión de bondad"15 de los segundos. J. Habermas, al igual que K.-O. Apel, se refiere al tema en la Ética del discurso al indicar que el error consistió, al menos, en pretender

12 Ibid., n. 29, p. 46. Como una "expansión e intensidad de vida". Spencer, un evolucionista darwinista, no advierte que la vida, como "vida humana", es criterio de verdad práctica, no inmediatamente de bien, porque le falta la participación libre y simétrica consensual de todos los afectados, que permite que la acción tenga pretensión de bondad (goodness claim). El "bien", que nunca se puede predicar de ningún acto absolutamente (se necesitaría una razón judicatoria absoluta, perfecta, que es imposible) es una síntesis compleja entre el aspecto material (que hace referencia a la vida humana) y el formal (la libre participación consensual) que operan como marcos o condiciones de posibilidad de la acción futura, temas de los caps. 1 y 2 de mi Ética de la liberación (1998). Moore no podía superar una simplificación extrema e ingenua de la temática propia de su época.

13 Ibid., cap. 3, nn. 336 ss., pp. 59 ss.: "Nothing is good but pleasure" (p. 61).

14 Sobre los diversos tipos de pretensión véase en mi Ética de la liberación, 1998, índice de temas: pretensión (p. 651).

15 Este tema, la "pretensión de bondad" (goodness claim, Anspruch-gut-zu-sein), deberé exponerlo en un trabajo futuro. En efecto, a la pretensión de validez (intersubjetiva), de verdad (referencia a la realidad siempre mediada lingüística, discursivamente), de rectitud (con referencia a los valores), de sinceridad (con referencia a las intenciones del hablante), etc., habrá que agregarle una nueva: cuando el agente cumple un acto, u organiza una institución, etc., habiendo cumplido las "condiciones de posibilidad" éticas (al menos los seis principios descritos en mi Ética de la liberación, 1998), decimos en ese caso que ese actor y su acto, institución, etc., tiene "pretensión de bondad". No se puede predicar de ningún sujeto ético, de ningún acto o institución, el que sea "bueno" (o un "bien") de manera perfecta, porque el acto es bueno por sus consecuencias, también a largo plazo (in the long run) y, absolutamente, "hasta el fin de la historia", y esta predicción es imposible de efectuar. Se necesitaría, analógicamente a como argumenta Popper contra la "planificación perfecta", poseer una inteligencia práctica infinita a velocidad infinita. Como esto es imposible, como no puede haber ningún sujeto o acto, institución, etc., perfectos, sólo se puede tener una honesta, seria, recta, sincera "pretensión de bondad". Es una nueva manera de dejar abierta la puerta a la universalidad de las condiciones (y principios éticos) del bien y a la incertidumbre de las decisiones y acciones concretas, dada la finitud humana. El "bien" es ahora perfectamente definible. Además el sujeto, el actor, la norma, el acto, la institución, etc., pueden tener "pretensión de bondad". 
juzgar a los enunciados descriptivos y normativos con las mismas reglas de objetividad. Su diferencia consistiría en que "las pretensiones de validez normativas mediatizan evidentemente una dependencia recíproca entre el habla y el mundo social, que no existe en cambio en la relación entre habla y mundo objetivo", ${ }^{16}$ propia de los enunciados empíricos o fácticos.

\section{LA "FALACIA NATURALISTA" EN LA LÓGICA FORMAL ${ }^{17}$}

En la lógica formal se afirma que de una premisa no se puede deducir analíticamente algo que no esté ya contenido en ella. De manera que para obtener una conclusión normativa ya debe encontrarse en las premisas dicha normatividad. Desde Frege, Carnap, Russell, el primer Wittgenstein, Stevenson, Hare o Ayer, esto es algo adquirido. No creo que haya que dedicarle espacio a repetir lo ya expuesto por dichos autores. Pero lo que no es incontrovertible es que los enunciados normativos sean sólo juicios de valor, y que éstos sean sólo juicios "subjetivos", faltos de objetividad, y que por ello no puedan ser integrados dentro de argumentos que obtengan validez de enunciados científicos, tal como cuando Ayer escribe: "Los juicios de valor [...] en la medida en que no son científicos, no son, literalmente hablando, significativos, sino que son simples expresiones de emoción que no pueden ser verdaderas ni falsas." 18

En este caso, no sólo no pueden deducirse enunciados éticos (ought to be) de enunciados fácticos (to be), sino que el nivel normativo mismo ha sido destituido de toda objetividad racional, con lo cual aún la posición valorativa de Moore ${ }^{19}$ queda confinada a una "subjetividad" donde los valores

16 Habermas 1983, p. 65. En nuestro caso la cuestión es más compleja, porque distinguiremos entre pretensión de verdad práctica y pretensión de validez propiamente dicha (véase Ética de la liberación, pp. 194 ss.).

17 Véase entre otros Frankenna 1939, Ayer 1952, Rabossi 1979, Stuhlmann-Laeisz 1983. Y antes aun Russell 1935, Carnap 1935, cap. 11; Stevenson 1944, pp. 37-93; Hare 1952, pp. 29-44.

18 Ayer 1952, pp. 102-103, en el cap. sobre "Critique of Ethics and Theology": "We shall set ourselves to show that in so far as statements of value [...] are not scientific, they are not in the literal sense significant, but simply expressions of emotion which can be neither true nor false."

19 Moore escribe casi al final de su Principia ethica: "I have now completed such remarks as seemed most necessary to be made concerning intrinsic values" (n. 134, p. 222). ¿Cuál puede ser la entidad ontológica de esos "valores intrínsecos"? Aquí estamos en la más pura axiología, semejante a la de Brentano (véase Ibid., p. x, n. 1), a la de Max Scheler o Edmund Husserl. Hablar de una axiología con "valores intrínsecos" es estar practicando una metafísica idealista (la de los valores), que buena parte de la metaética analítica posterior supuso sin deconstruir. El mismo Heidegger, en la Carta sobre el humanismo escrita a Sartre, es más escéptico acerca de los valores que muchos lógicos que aplican las reglas de la lógica formal a 
están faltos de toda consistencia "objetiva" (e intersubjetiva). Sin embargo, esa posición extrema ha sido revisada, y los juicios de valor han obtenido al menos en el presente una objetividad consensual, intersubjetiva, por referencia a una comunidad histórica, cultural, pragmática de los hablantes. Por otra parte, mi posición personal sobre el tema es mucho más compleja, ya que pretendo argumentar a partir de un cierto tipo específico de juicios de hecho o fácticos que contienen implícitamente alguna normatividad. ${ }^{20}$

A los fines de estas reflexiones nos resta indicar que la "falacia naturalista" se sitúa adecuada y estrictamente como falacia argumentativa (en Moore se hablaba en cambio de una falacia atributiva o predicativa) denunciada por la lógica formal analítica, y en el nivel discursivo de esta lógica.

\section{IV. ¿SE PUEDE “EXPLICITAR" PRÁCTICO-MATERIALMENTE DESDE EL "SER" EL "DEBER-SER"? 21}

Se intentará mostrar si es posible descubrir contenidos normativos en ciertos juicios empíricos. Gracias a esto se podría intentar manejar argumentativamente juicios de hechos o enunciados descriptivos (que contienen normatividad), como premisa mayor, cuya premisa menor fueran juicios empíricos de conclusiones científicas (de las ciencias sociales), a fin de llegar a inferencias normativas o éticas. Esto es de la mayor importancia, porque se podrían desarrollar argumentos que no caigan en la falacia naturalista, tanto en la fundamentación de los principios éticos como en la aplicación de dichos principios en las llamadas "éticas aplicadas" o diferenciales, tan necesarias en los diversos campos de la ciencia o de las profesiones o funciones sociales.

Sería necesario, en primer lugar, tratar de encontrar un tipo de enunciados (que puedan operar como premisa mayor) que sean al mismo tiempo descriptivos o de hecho ("ser") y normativos ("deber-ser") —no necesaria-

estos "seres fantasmagóricos", si se los piensa no integrados como mediaciones en estructuras prácticas concretas (y retenidas memorativamente en ciertas partes del cerebro humano). Como seres independientes "en sí", a los valores se les debería asignar una existencia en algo así como un espacio o "kosmos noetós" à la Platón. Véase en mi Ética de la liberación, tesis 12, p. 623; y en el vol. 1 de mi obra Para una ética de la liberación latinoamericana (Siglo XXI, Buenos Aires, 1973), la "valoración" de las "posibilidades" en un sentido heideggerianonietzscheano. Los "valores" no pueden ser fundamento de la ética, ni pueden jamás tener ninguna existencia real independiente; en último caso los fines, de los cuales los valores son una cualidad de sus mediaciones, tendrían la función de ser fundamento. Pero tampoco, porque es la vida humana la que "pone" dichos fines.

20 Véase mi Ética de la liberación, cap. 1.

21 Véase Höffe 1988, MacIntyre 1981, Brandom 1994, Habermas 1999, cap. 3. 
mente como juicio de valor. Deberían referirse, en el nivel material, ${ }^{22}$ a la "vida humana" en cuanto humana, por ejemplo:

1. El ser humano es un ser viviente.

2. Juan es un ser humano, y tiene por ello cerebralmente conciencia, autoconciencia y responsabilidad sobre su vida.

3. Cuando Juan tiene hambre intenta producir y reproducir su vida comiendo.

4. Para seguir siendo un viviente responsable, Juan debe comer.

La vida es el modo de la realidad ${ }^{23}$ humana. Es la condición absoluta de la existencia del ser humano. La argumentación ética se liga materialmente a este tipo de enunciados descriptivos que tienen contenidos implícitos normativos. Y esto porque el ser humano viviente es autorreflexivo, ${ }^{24}$ es decir, es "responsable" $25 \mathrm{de}$ /por su propia vida (ya que el cerebro humano tiene la capacidad de cumplir las funciones neurológicamente superiores de categorización perceptual y conceptual, memoria, capacidad lingüística, conciencia, y, gracias a todo ello, alcanza la "autoconciencia" de su propia vida, y de sus actos). Repito: la "responsabilidad" sobre la vida misma es la condición de posibilidad de la normatividad como tal (que puede ser enunciada por juicios descriptivos de contenido normativo). Pueden igualmente enunciarse juicios sobre actos, descriptivos, empíricos, como en el caso del juez, cuando "basado en hechos", puede juzgar un acto concreto como si se hubiera obrado "con responsabilidad" o "plena conciencia" (meritoria o culpable). Hay entonces en la premisa empírica o descriptiva un contenido al mismo tiempo ético, normativo.

22 El argumento habría que repetirlo en los niveles formal, de factibilidad y crítico. Cada principio ético permite superar de esta manera la falacia naturalista. En Ética de la liberación, me refiero a seis principios.

23 Esta expresión la tomamos de Zubiri, por lo que nos llama la atención que Jordi Corominas 2000, pp. 74 ss., nos critique por exponer una posición vitalista reductivista. Afirmamos la vida humana en el mismo sentido que Zubiri lo hace en sus libros (tema que Corominas no toca en la obra del filósofo madrileño, y al que éste le dedica largas páginas) y aun Ellacuría.

24 Véase en la obra del premio Nobel de neurología, G.M. Edelman (1989 y 1992) el tema de la "autoconciencia".

25 Este tema merecería un tratamiento específico, ya que algunos niegan la existencia de una tal responsabilidad, pero en este caso todo el ámbito del derecho civil y del derecho penal quedaría sin objeto. Sin embargo, estamos hablando de algo más radical, de la "responsabilidad" sobre la propia vida (que podría ser llamada "metafísica" o ética en el sentido levinasiano). Esta responsabilidad (ético-metafísica) es anterior a la responsabilidad (óntica) sobre los actos; aquélla es el presupuesto, es el "momento" sobre el que se construye todo el sistema del derecho, de la vida política y de las relaciones humanas más cotidianas del sentido común. 
Esa normatividad puede ser "implícita". Pero se puede igualmente "explicitar" (lo implícito), y esto sería un "fundamentar dialécticamente" prácticomaterialmente, ${ }^{26}$ no por deducción lógico-formalmente, juicios normativos de juicios de hecho.

La noción de "fundamentar" significa ahora "explicitar" lo "implícito". Así lo entendían dialécticamente Hegel o Marx, para quienes la "esencia" incluía implícitamente al "aparecer" fenoménico. La "forma" fenoménica con la que aparece una mesa (por ser la mesa del maestro en el aula) se "funda" (o explicita una posibilidad) en el "todo" funcional (o "esencia" para Hegel) al que sirve. La mesa del maestro tiene "otra forma" diferente de la de la mesa del carpintero o la de un comedor. Las diversas "formas" de la mesa son "explicitación" (o se "fundan") en las "esencias" o "totalidades" (y por ello es fundamentación dialéctica) diversas (el acto de enseñar es diferente del de hacer muebles o de comer; todos ellos establecen "totalidades" funcionales diversas e implícitas, que determinan las diferentes "formas" con las que aparecen las mesas). El ser viviente humano, como hemos dicho, "incluye" a la autoconciencia y "responsabilidad" sobre su vida misma, como una determinación propia (implícita en todo enunciado sobre el ser humano como humano), que se presupone (y que funda por su parte cuando es explicitado) en toda otra dimensión humana como humana, y por ello como "deber": "deber" sobre todo aquello acerca de lo que se tiene responsabilidad, ${ }^{27}$ porque es necesario para la vida - y también para la vida cultural en la cual se haya inmerso, pero en este caso como un deber con referencia a "valores", que es ya otro tema.

El "pasaje" del contenido empírico al normativo no se habría dado, sin embargo, entre 3. y 4., sino que "implícitamente" 28 en 1. ya tendríamos contenidos normativos al referirnos al "ser humano". Y esto porque el "ser humano" como tal es ya un "sujeto ético", es "responsable" de su vida, y queda "obligado" también (ésta es la normatividad "oculta" del juicio empí-

26 "Materialmente" quiere indicar que no se trata de juicios analíticos, propios de la mera lógica formal, sino de juicios empíricos con referencia a la vida humana, sustantivos, y que pueden incorporar mayor complejidad en su contenido.

27 Se es responsable "ante" el tribunal (en primer lugar de la propia conciencia ética) "por" lo conducente o no a la sobrevivencia de la propia vida. Se "tiene" responsabilidad acerca de la propia vida y por ello "obligación" ética (deber) sobre los medios para su sobrevivencia.

28 Nos inspiramos ahora en la noción de "implícito" tanto de Höffe (1988) como de Brandom (1994). Las "reglas" están implícitas en todo ejercicio pragmático de la lengua, en un estado pre-predicativo, pero pueden "hacerse explícitas" (Making It Explicit es el título del libro de Brandom). 
rico $^{29}$ ) al querer vivir, o al menos a seguir viviendo. ${ }^{30} \mathrm{El}$ que no quiere vivir no queda desligado de esta "obligación", simplemente no la cumple y puede fácticamente hacerlo, pero contradictoriamente. Todas estas determinaciones son ya siempre normativas "implícitamente". En ética se trata entonces de "explicitar" lo "implícito" y no de deducir lo normativo de lo empírico. El enunciado empírico sobre el ser humano como humano es siempre ya "implícitamente" normativo.

Por otra parte, el "querer", ${ }^{31}$ del "si se quiere ${ }^{32}$ vivir", es un momento ya fundado. El "ser-responsable" por la propia vida no puede elegirse ni ser anticipado. Nadie tiene derecho a la vida (sólo se tiene derecho a la sobrevivencia), porque tendría que ser un sujeto de derecho antes (en el tiempo) de existir como viviente. El viviente humano se encuentra "desde siempre" - desde que es concebido en el seno materno- como existente con vida, como viviente, y como irremediablemente responsable por su propia vida. Desde nuestra gestación "la vida nos ha sido dada a cargo", decía bellamente Ernst Bloch. ${ }^{33}$ Que se ejerza posteriormente ónticamente esa responsabilidad, de una u otra manera, es un momento secundario y ya fundado. No se puede ser responsable por la propia responsabilidad originaria con respecto a la vida propia. Se puede de muchas maneras ejercer esa responsabilidad abismal y primera. El "querer" o "no querer" vivir son dos modos del cumplimiento del deber inevitable del responsable por su vida. El que quiere vivir lo hace responsablemente. El que "no quiere vivir" cumple la irresponsabilidad primera (con referencia a su responsabilidad

29 Este estado de "oculto" (implícito) es a lo que se refiere Otfried Höffe cuando escribe que "la mediación de un elemento normativo y del elemento descriptivo" (Höffe 1988, p. 134). Lo que aplicado a una filosofía del derecho se enunciaría como la exigencia de un "principio de moralidad" (premisa mayor), con una "condición de aplicación de justicia" (premisa menor), y un "principio de justicia" (conclusión), lo que permitiría desarrollar la "estructura fundamental de un derecho natural que no cae en la falacia naturalista" (ibid.), expone Höffe.

30 Un padre está obligado a seguir viviendo, no sólo con respecto a su propia vida bajo su responsabilidad, sino que, además, al haber puesto en la existencia viviente a su hijo, ha adquirido la responsabilidad con respecto al pleno desarrollo de la vida de éste, y por ello tiene deberes que cumplir para con su hijo, y de la misma manera con referencia a otros deberes comunitarios.

31 Y nos referimos a la objeción de Corominas 2000, pp. 74 ss.

32 El mero "querer" no tiene aquí ningún contenido particular, sino que es una reafirmación posterior, "óntica" la denominaría Heidegger, de la misma vida humana en cuanto asumida reflexivamente (en acto segundo) desde la Voluntad-de-vivir (Wille zum Leben) ontológico en el sentido de Schopenhauer, Freud o el neurólogo Antonio Damasio (1994). El "prometer" de John Searle (1964) agregaba al acto el contenido normativo propio de la "promesa", que especifica un contenido performativo innecesariamente, porque el acto humano como humano es ya implícitamente normativo. Se trataría de lo que MacIntyre (1969, p. 46) indica cuando escribe que Hume fundamentó la ética "in human needs, interests, desires, and happiness".

33 Véase mi Ética de la liberación, cap. 5.4. 
primera, diría Levinas, que además es responsabilidad ante los otros y antes que sólo ante sí mismo, porque la misma vida humana la hemos recibido de una comunidad que nos antecede y con respecto a la cual somos deudores, aunque no paguemos irresponsablemente la deuda). Repito: el "poder no querer vivir" -que anticipa al suicidio- es ya un acto posible desde el horizonte ético. Y el que se suicida, puede fácticamente hacerlo, pero no puede hacerlo éticamente fundado, o, de otra manera, no es una expresión responsable de su radical responsabilidad sobre su propia vida en vista de la ajena. Es un acto ónticamente irresponsable (e irracional por ello) como negación de la responsabilidad originaria, ética, o "metafísica", en el sentido anotado más arriba. De otra manera: se "quiere" ahora y aquí, y como un acto de la voluntad que se expresa por estados corporales o por juicios comprensibles a cualquier oyente. Pero, al mismo tiempo, el "querer" tiene ya un cierto contenido normativo, porque el querer del "ser humano" incluye reflexividad, autoconciencia, y por ello referencia a la "responsabilidad" primera sobre su vida, y por ello sobre sus actos. "Lo que" (la finalidad o el objetivo) se quiere en última instancia es la vida (y la permanencia de ella como "sobrevivencia"), que por su parte tiene exigencias u obligaciones $^{34}$ con respecto a las mediaciones de la efectiva sobreviviencia, como el comer (mediaciones o exigencias que son jerárquicamente categorizadas evaluativamente por el cerebro y memorizada, también en un nivel óntico en la tradición de los "valores" de la propia cultura). Por ello, si se quiere vivir es necesario comer. Pareciera que esa "necesidad" no es todavía una "obligación" normativa. Pero que el ser humano, como hemos dicho, sea un ser autorreflexivo, responsable de su propia vida, significa que tiene "a su cargo" su propia vida. La "necesidad" física de comer (como puede hacerlo un animal) es doblada por el ser reflexivo y responsable (por un "tenerse-a-cargo": re-sponsable, que responde "por" sí mismo "ante" sí mismo y los otros) como "obligación" ética en tanto referencia última a un sujeto autónomo que en su ser libre "debe" cuidar de su vida.

34 Kant mostraba cómo la ley o norma de la voluntad se impone a ésta no con la necesidad de una ley física, sino como una "obligación" o un imperativo que supone la libertad. El "querer" y el "deber" impera no con una determinación del tipo de la ley física. Además, agrego ahora, el muy particular "querer vivir" es la condición absoluta de todo otro "querer" y se imponen gracias a la responsabilidad (sobre la vida) como "obligación" primera, que funda todas las "obligaciones" restantes. El que "no-quiere-vivir" deja de estar "obligado" en general. De la misma manera el que se suicida usa irresponsablemente su vida al querer eliminarla. Es un acto contradictorio: se actúa para eliminar toda posibilidad futura de actuar. Aunque contradictorio es posible, pero no éticamente posible. Y es contradictorio performativamente cuando se enuncia: “-iActúo para no actuar nunca jamás!". Es performativamente contradictorio porque todo acto tiene una finalidad. Pero este acto elimina toda finalidad posible; tiene como finalidad la no-finalidad absoluta. 
Se podría por ello continuar el argumento siguiendo una vía negativa:

5. Si 1. y 2.;

6. entonces, si Juan no quisiera vivir, en último término se suicidaría;

7. al ser el suicidio un acto contradictorio, ya que es el acto que elimina toda posible actuación futura (empíricamente alguien puede suicidarse, pero no puede justificarlo ni practicarlo éticamente, ni fundarse sobre él un orden ético o instituciones);

8. luego, Juan, como racional y responsable, no debe suicidarse, es decir, debe intentar por todos los medios producir y reproducir su vida.

La negación de la vida en el suicidio, como lo expresaba claramente Wittgenstein, es negación de toda obligación ética, es decir, de la ética en cuanto tal; o, de otra manera, "si el suicidio está permitido, todo está permitido" (lo cual no indica que el suicidio sea bueno o malo, sólo afirma que es posible fácticamente pero éticamente contradictorio). Siendo la vida la condición absoluta del sujeto ético, el suicidio deja sin existencia al sujeto y a la ética.

Pasando a otro tema, se puede hacer otro uso del argumento al incluirse una premisa menor (siendo la premisa mayor 1. y 2.) que fuera una conclusión científica, como por ejemplo en el siguiente enunciado de una posible economía política crítica:

9. El sistema capitalista produce masas de pobres (como lo prueba Amartya Sen; el pauperismo atenta contra la vida o contra aspectos esenciales de la vida de ciertos seres humanos, las víctimas). Juan es uno de esos pobres.

10. Si Juan es responsable de su vida que tiene que vivir según las exigencias estándares del momento histórico, es decir, dejar de ser pobre,

11. entonces, Juan debe luchar contra (o al menos transformar) el sistema capitalista.

En el lugar de la premisa 9. (que es la premisa menor con respecto a los juicios empíricos 1. y 2.) se pueden colocar otras conclusiones de las ciencias que de alguna manera se refieren en última instancia a algún aspecto de la vida humana. La ética, en su nivel material entonces, podría inferir conclusiones normativas de toda conclusión científica de las ciencias sociales críticas al incorporar dichos enunciados empíricos (si, como hemos dicho, pueden ser relacionados con momentos necesarios de la vida humana), efecto de la racionalidad científica o explicativa, dentro de la discursividad ética.

Se trata entonces de prestar atención al hecho de que el "contenido normativo implícito", en el enunciado descriptivo que desempeña la fun- 
ción de premisa mayor (1. y 2.), o menor (3., 6., 10.), incluye un componente "oculto" ya normativo. En especial, el enunciado normativo de la conclusión "fundada" (11.) subsume las conclusiones de las ciencias (9.): "explicita" entonces un contenido normativo "implícito" en las premisas.

Habría todavía otras maneras de alcanzar un resultado semejante. H. Putnam ${ }^{35}$ muestra que los juicios de valor son constitutivos de los mismos juicios empíricos científicos (por ejemplo, la claridad, la simplicidad, el orden, etc. que el discurso científico presupone responden a juicios de valor). Charles Peirce hablaría todavía del "socialismo lógico" que opera como un presupuesto ético necesario de toda comunidad científica, posición desarrollada por Karl-Otto Apel de manera creativa y actualizada. ${ }^{36}$ En estos casos, el mismo juicio científico o la comunidad científica presupondrían actitudes que pueden enunciarse en juicios éticos o normativos, que desempeñan la función de principios constitutivos de la misma argumentación, como el reconocimiento del otro argumentante como igual, es decir, afirmado como simétrico y de equivalente dignidad. ${ }^{37}$ Así, la ética dejaría de ser un discurso subjetivo, meramente opinable y opuesto al discurso científico. Sería un discurso diferenciable, que se sitúa en otro nivel, pero objetivo ${ }^{38}$ y racional (o tan poco racional para Putnam) como el científico. No más racional, pero tampoco menos racional, sino situado en otro nivel de objetividad intersubjetiva, o en un tipo de racionalidad más consciente de la falibilidad ${ }^{39}$ (y falsabilidad) de sus enunciados: la incertidumbre propia de lo ético concreto. El tiempo del dogmatismo positivista ha pasado, y la ética tiene mayor posibilidad de mostrar su función histórica impostergable ante una humanidad en riesgo de cometer, por falta de conciencia, objetividad y racionalidad ética, un suicidio colectivo (ecológico, económico, político, militar, etcétera).

Pienso que ahora estaríamos en el camino de evitar cometer la "falacia naturalista", al situar la cuestión en un horizonte concreto, de mayor com-

35 Véase por ejemplo el tema en Putnam 1994.

36 Véase Apel 1973, t. 2, Segunda parte.

37 Apel indica que el que argumenta ya presupuso siempre a priori que el otro argumentante tiene igual dignidad como persona. Este reconocimiento previo es ético (como acto de una "razón ético-orginaria"; véase el tema en mi Ética de la liberación.

38 Aunque con un tipo propio de "objetividad" propia de la intersubjetividad de la comunidad práctica, social, histórica.

39 La "pretensión de bondad" de todo acto ético es por definición corregible, falsable, pero no por ello menos honestamente pretendido en su bondad - que me exige, a partir de la corrección o falsación, cumplir o transformar el acto en el sentido exigido de la indicada y anterior "pretensión de bondad". 
plejidad práctico-material o dialécticamente. La ética podría ahora además subsumir las conclusiones de la ciencias empíricas y construir desarrollos nuevos en lo que pudiera llamarse "éticas aplicadas": la ética de la medicina, de la tecnología, de la economía, de la administración, del periodismo, etc., etc., en las que integraría como premisa menor las pertinentes y mejores conclusiones de cada ciencia mostrando las exigencias deónticas que ellas presuponen y que de ellas se concluyen. Dichas exigencias no son sólo intrínsecas a cada disciplina o campo profesional (como las desarrolladas por una ética que el ejercicio de la medicina pide como condición de posibilidad del propio "sistema de la salud", por ejemplo, al no quitar indebidamente un cliente a otro médico u hospital), sino guardando exterioridad, o como el poder juzgar a dicho "sistema" como totalidad desde la alteridad de la vida humana. Sería, por ejemplo, un poder criticar éticamente, desde la vida humana y desde una reconstrucción crítica del concepto de "enfermedad", el modo como el sistema capitalista de la salud (fetichizado por el uso monopólico de la ciencia como "saber" sanar y de la tecnología desarrollada - monopólicamente en manos de pocas corporaciones trasnacionales-como única mediación para la salud) "explota" al enfermo económicamente, convirtiéndolo en un "cliente obligado" (víctima alienada e inocente) y absolutamente dependiente, como mera mediación para permitir el aumento de la tasa de ganancia de la industria farmacéutica, de los sistemas de instituciones privadas de la salud (clínicas, sanatorios, hospitales), del gremio autoprotegido de médicos como los únicos conocedores monopólicos del Poder de "sanar" la "enfermedad", que hace años Ivan Illich comenzó a criticar tan atinadamente, lo mismo que Michel Foucault -microsistemas autorreferentes fetichizados. Ética y ciencia podrían cumplir así una labor diferenciada pero articulable, y, además, los principios éticos podrían fundarse (explicitarse) desde enunciados descriptivos "de vida humana" (que incluirían aspectos normativos).

BIBLIOGRAFÍA

Apel, Karl-Otto (1973), Transformation der Philosophie, Suhrkamp, Fráncfort, t. 1-2. Atkinson, Ronald (1969), "Hume on Is and Ought", en Hudson, 1969, pp. 51-58. Ayer, Alfred Jules (1952), Language, Truth and Logic, Dover Publications, Nueva York.

Beck, Lewis White (1974), "What Must Be and Is-Ought in Hume", Philosophical Studies, no. 26, pp. 219-228. 
Blegvad, Mogens (1963), "Mill, Moore and the Naturalistic Fallacy", en Helge BrattSten Dunér et al., Philosophical Essays, dedicada a Gunnar Aspelin, CWK Gleerup Bokförlag, Lund, pp. 9-19.

Brandom, Robert (1994), Making it Explicit, Harvard University Press, Cambridge. Camps, Vitoria (1976), Pragmática del lenguaje y filosofía analítica, Península, Barcelona.

Carnap, Rudolf (1935), Philosophy and Logical Syntax, Tubner y Co., Londres. [Traducción española: Filosofía y sintaxis lógica, 2a. ed., trad. de César Molina, Universidad Nacional Autónoma de México-Instituto de Investigaciones Filosóficas, 1998.]

Corominas, Jordi (2000), Ética primera, Desclée de Brouwer, Sevilla.

Damasio, Antonio (1995), Descartes' Error, Emotion, Reason, and the Human Brain, A. Grosset, Nueva York.

Dubais, P. (1967), Le problème moral dans la Philosophie anglaise de 1900 à 1950, Vrin, París.

Dussel, Enrique (1998), Ética de la liberación en la edad de la globalización y la exclusión, 3a. ed., Trotta/uam, Madrid/México, 2000.

Edelman, Gerald M. (1989), The Remembered Present. A Biological Theory of Consciousness, Basic Books, Nueva York.

_-(1992), Bright Air, Brillant Fire. On the Matter of the Mind, Basic Books, Nueva York.

Frankena, William K. (1939), “The Naturalistic Fallacy”, Mind, no. 48, pp. 464-477. Hare, Richard M. (1952), Language of Morals, Oxford University Press, Oxford.

Habermas, Jürgen (1983), Moralbewusstsein und kommunikatives Handeln, Suhrkamp, Fráncfort.

—_(1999), Wahrheit und Rechtfertigung, Suhrkamp, Fráncfort.

Höffe, Otfried (1988), "Droit naturel sans paralogisme naturaliste", en L' etat et la justice, Vrin, París, pp. 109-139.

Hudson, William D. (1969), The Is-Ought Question, MacMillan, Bristol.

Hume, David (1958), A Treatise of Human Nature, L.A. Selby-Bigge (ed.), Oxford University Press, Oxford.

Hunter, G. (1962), "Hume on Is and Ought", Philosophy, abril.

Kutschera, F. von, "Das humesche Gesetz", Grazer philosophische Studien, no. 4, pp. 1-14.

Lewy, Casimir (1964), "G.E. Moore on the Naturalistic Fallacy", en Proceedings of the British Academy, vol. 1, Oxford University Press, Londres, pp. 251-262.

MacIntyre, Alasdair C. (1964), "Hume on Is and Ought", en Hudson, 1969, pp. 3550.

- (1981), After Virtue, Notre Dame University Press, South Bend.

Moore, George Edward (1903), Principia ethica, Cambridge University Press, Cambridge (Reino Unido). [Traducción española: Principia ethica, 2a. ed., trad. de Adolfo García y Ana Isabel Stellino, Universidad Nacional Autónoma de MéxicoInstituto de Investigaciones Filosóficas, 1997.]

Putnam, Hilary (1994), Words and Life, Harvard University Press, Cambridge. 
Rabossi, E. (1979), Estudios éticos: cuestiones conceptuales y metodológicas, Universidad de Carabobo, Valencia (Venezuela).

Russell, Bertrand (1935), Religion and Science, Nueva York.

Sádaba, J. (1989), "Ética analítica", en V. Camps (ed.), Historia de la ética, Crítica, Barcelona, t. 3, pp. 153-176.

Schilpp, Paul (1942), The Philosophy of G.E.Moore, Northwestern University, Evanston.

Searle, John (1964), "How to Derive Ought from Is", en Hudson, 1969, pp. 120-134.

Stevenson, Charles (1944), Ethics and Language, Yale University Press, New Haven. (1963), Facts and Values, Yale University Press, New Haven.

Stuhlmann-Laeisz, Rainer (1983), Das Sein-Sollen Problem, Stuttgart.

Recibido: 28 de octubre de 2000 . 